\title{
INCONSISTENT VISUAL ANALYSES OF INTRASUBJECT DATA
}

\author{
ANThony DeProspero and Stanley Cohen
}

\author{
WESTON HOSPITAL AND WEST VIRGINIA UNIVERSITY
}

\begin{abstract}
Visual inspection has been the method of analysis most widely employed to evaluate the functional control demonstrated by any given set of intrasubject replication data. To identify the influence of certain graphic characteristics on these evaluative behaviors, 36 "ABAB reversal" figures were constructed. They were sent to 250 reviewers of behavioral journals. Their evaluation of each figure was expressed as a rating on a 100point scale of "experimental control." Mean interrater agreement was 0.61. In addition to this rating, a verbal description of evaluation criteria was requested. It was also found that graphic characteristics determine evaluative judgments in concert rather than singly. For example, phase mean changes had to be a pattern consistent with the hypothesized effect of the experimental variables, while degrees of mean shift and variability were less important. A description of the following evaluative criteria was presented: (a) topographic characteristics, (b) format of data presentation, (c) intraexperimental, and (d) extra-experimental circumstances.

DESCRIPTORS: visual analysis, methodology, judgmental aids, reliability, evaluative criteria
\end{abstract}

The main problem for analysis of intrasubject data is to determine whether changes in behavioral scores follow changes in experimental conditions with sufficient regularity to warrant the conclusion that experimental control has been demonstrated. The behavior analyst has typically employed visual inspection of the data as a means for doing so (Kazdin, 1976). Discussions of visual interpretation (Hersen and Barlow, 1976; Sidman, 1960) have identified at least four graphic features which are of interest to researchers evaluating intrasubject data. These are: (a) mean shift across phases in relation to experimental hypotheses, (b) magnitude of mean shift across phases, (c) score fluctuation or variation within phases, and (d) slope of data in relation to experimental hypotheses.

Portions of this paper were presented at the ninth annual meeting of the Association of Advancement of Behavior Therapy, New York, 1976. The authors would like to thank W. Stewart Agras for his assistance in conducting the survey and Don Hake for his careful comments and editing on an earlier draft. Reprint request should be addressed to Anthony DeProspero, Psychology Service, Weston Hospital, Weston, West Virginia 26542.
There exists evidence that visual inspection is not entirely satisfactory. Consider the work of Jones, Weinrott, and Vaught (1978) who selected a set of 58 pairs of adjacent phases from graphs depicting "nonobvious" experimental results from the Journal of Applied Behavior Analysis. A panel of 11 judges showed greater agreement in evaluating meaningful changes in level for adjacent phases that were not statistically reliable by time-series analysis than for phases that were statistically reliable. This "suggests that statistically reliable experimental effects may be more often overlooked by visual appraisals of data than nonmeaningful effects" (Jones et al., 1978, p. 280). Over all pairs of phases, the median intercorrelation of the judges' agreement with themselves was only .39. A particular property of the data (serial dependency) was found to affect adversely the agreement of the judges.

Another example of interpretive inconsistency was found by White (1971) who demonstrated that individuals can vary widely in their interpretation of graphed data, even to the extent that some would interpret a trend as being "ac- 
celerating" while others judged the same trend to be "decelerating." In practice, therefore, such inspection can lead to unequivocal conclusions only when the behavior change is so dramatic that no critic would disagree with its significance. While it may be desirable to have judgmental errors on the "conservative" side for some purposes (Baer, 1977), this state of affairs could conceivably result in inefficiency of experimental effort. For example, unfavorable review about nondramatic results might lead to a variable being dropped prematurely from experimentation. This purpose of the present study was to determine whether agreement in visual judgment could be reliably attributed to certain features of the graph.

\section{METHOD}

\section{Stimulus Materials}

In order to represent those characteristics thought to influence visual interpretation, a set of simulated "ABAB reversal design" graphs were constructed. Those graphs illustrated the four factors thought to represent characteristics important for visual analysis.

The first of the graphic factors (pattern of mean shift) was represented by three patterns of mean shifts across phases: (a) an "ideal" pattern of results with consistent increases in both " $B$ " phases and an intervening return to baseline (top portion of Figure 1A), (b) "inconsistent treatment" pattern with no mean change in the first three phases, concluding with an acceleration in the last phase (middle portion of Figure $1 \mathrm{~A}$ ), and (c) an "irreversible effect" pattern of acceleration in the second phase with no subsequent mean change (bottom portion of Figure $1 \mathrm{~A})$.

The second characteristic was called degree of mean shift across phases. It is simply the percentage of mean change from phase $\mathrm{X}$ to phase $\mathbf{X}+1$. To illustrate how this was computed, consider two experimental phases, a baseline with a mean of 10.0 and a treatment period with a mean of 12.5 . The difference (2.5) would be divided by the mean of the earlier phase (10.0) to yield a value of .25 . All three mean shifts in an "ABAB" graph were equal, and three values were considered: (a) 1.0 (top portion of Figure 1A), (b) .50 (top portion of Figure 1B), and (c) .25 (bottom portion of Figure 1B).

The third characteristic, score fluctuation or variation within phases, was manipulated by selecting "behavior scores" with a particular relationship between mean and standard deviation. To illustrate, consider a phase with scores that have a mean of 10 and a standard deviation of 1.0. The standard deviation is divided by the mean to yield a variability coefficient of .1. Two values were represented, .1 (top portion of Figure 1A) and .25 (top portion of Figure 1C).

The final graphic characteristic was concerned with "trend," or the extent to which the data fell along a line with a given slope. Two values were represented: one-half of the graphs had zero slope in all phases (Figure $1 \mathrm{~A}$ ), and onehalf had a linear slope of 30 degrees in the "positive" or "increasing" direction (bottom of Figure 1C).

These data were constructed in the following manner. First, 144 phase means were selected, four for each of the 36 "ABAB" graphs. The means were selected so that 12 pairs of phase means each illustrated the three patterns and degree of mean shift described above. This procedure resulted in four straight lines in each phase of the "ABAB" designs. For half the graphs, the "trend" was introduced by rotating these lines 30 degrees about the middle data point of each phase. To introduce the variability, a set of 1,440 deviation scores (one for each of the 10 points in each phase) was generated. These had a mean of 0 , and a standard deviation of 1.0. Each behavior score was then multiplied by the deviation score and the indicated variability coefficient. These operations resulted in randomly distributed variability that had no effect on the pattern or degree of mean shift, or on the slope. 


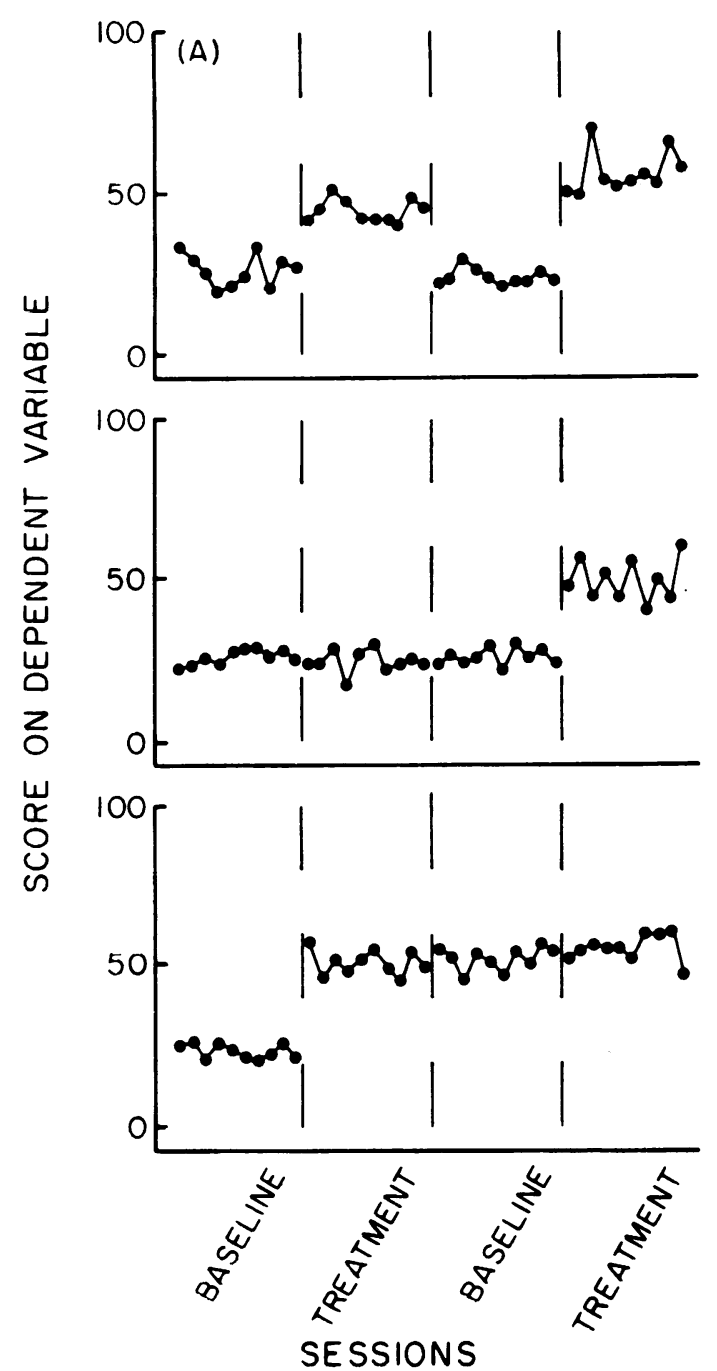

Fig. 1. Hypothetical data from simulated reversal design experiments. (A) Top: "ideal" pattern of results; middle: "inconsistent treatment" pattern of results; bottom: "irreversible effect" pattern of results. (B) Top: 0.5 degree of mean shift; bottom: 0.25 degree of mean shift. (C) Top: 0.25 in-phase variability; bottom: 30 degree slope in all phases.
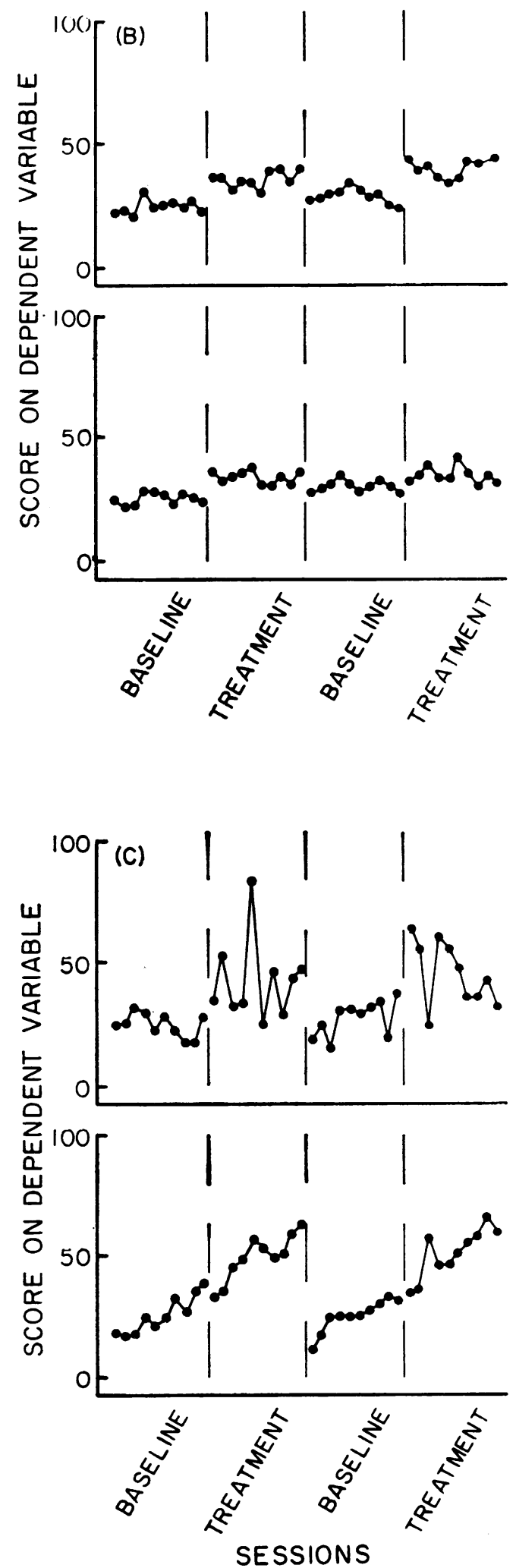


\section{Subjects}

A pool of 250 constituted the sample, 215 members of the Board of Editors or Guest Reviewers of the Journal of Applied Behavior Analysis and 35 members of the Board of Editors of the Journal of the Experimental Analysis of Behavior. At the end of six weeks, 114 replies had been received for a response rate of $43 \%$. Six respondents stated that they could not meaningfully answer the questions in the absence of circumstantial information, leaving a total of 108.

\section{Procedure}

The 36 graphs were divided into four sets of nine, each containing at least one sample of each of the three patterns of mean shift. One set was sent to each subject. The lower portion of the figures contained the question, "Using the following scale, how satisfactory a demonstration of experimental control do you consider this to be?" The scale was labeled "low" at the zero end and "high" at the 100 end. The average of all ratings given each figure was determined, and is hereafter referred to as mean rating. To permit the expression of idiosyncratic evaluative criteria, subjects were asked to list the factors they considered when analyzing graphic presentations of data, in general.

\section{RESULTS}

\section{Interrater Agreement}

To assess interrater agreement, the Pearson product moment correlation was calculated for each pair of raters who had reviewed the same set of graphs. The average correlation was .61 , with a standard deviation of .26 .

\section{Grapbic Characteristics \\ and Evaluative Criteria}

Table 1 presents graphic characteristics, size of rating sample, and the mean, standard deviation, and range of ratings for each graph. Pattern of mean shift was a critical characteristic in that mean rating fell off very rapidly for any pattern other than the "ideal." It appears that the minimum for respectable ratings was that the means of phases change in a pattern consistent with the hypothesized effect of the experimental variables. As one might expect, larger degrees of mean shift produced more favorable judgments, but the effect was not so dramatic. The bottom portion of Figure 1B has the same pattern of mean shift, variability coefficient, and a slope of trend as the top portion of Figure $1 \mathrm{~A}$, but the degree of mean shift is only one-fourth as large. Lower degrees of variability and the absence of trends produced more favorable judgments, but it is noted that these factors were influential only within the "ideal" pattern of mean shift; and, even here, an unfavorable combination of degree of mean shift and variability coefficient was sufficient to result in very low ratings.

It is apparent that each factor represents a source of strength in the judgment process, but it is equally apparent that the graphic factors exert their effects in concert. The mean rating for each of the 36 graphs was subjected to a four-way (pattern, degree, variability, slope) repeated measures analysis of variance. Pattern and degree of mean shift were highly significant, $F(2,935)=154.06$, and $F(2,935)=94.39$, respectively (in the sense of having a reliable effect upon mean rating). Together they account for only a small portion of the variance (eta squared $=.27$ ). Several of the interactions are significant as well, but these account for even less variance and are not reported.

\section{Verbal Statement of Evaluative Criteria}

The open-ended question about evaluative criteria prompted a comprehensive range of criteria employed by behavioral researchers. A certain amount of paraphrasing and categorization was done, but every effort was made to reflect the opinions as they were expressed, and in the appropriate context. The numbers in parentheses represent the frequency with which a given comment was made. In general, there were four "clusters" of statements. 
Table 1

Relationship of Graphic Characteristics to "Demonstration of Experimental Control"

\begin{tabular}{|c|c|c|c|c|c|c|c|}
\hline \multirow{2}{*}{\multicolumn{2}{|c|}{$\begin{array}{l}\text { Degree of } \\
\text { Mean Shift }\end{array}$}} & \multirow{2}{*}{$\begin{array}{c}\text { Witbin-Phase } \\
\text { Variability }\end{array}$} & \multirow{2}{*}{$\begin{array}{l}\text { Slope of } \\
\text { Trend }\end{array}$} & \multirow[b]{2}{*}{$N^{a}$} & \multicolumn{3}{|c|}{ Rating } \\
\hline & & & & & Mean $^{\mathrm{b}}$ & S.D. & Low/High b \\
\hline \multicolumn{8}{|c|}{ "IDEAL" PATTERN OF RESULTS } \\
\hline 1.0 & (Top, Figure 1A) & 0.1 & $0^{\circ}$ & 32 & 78.6 & 18.7 & $3 / 100$ \\
\hline 1.0 & (Bottom, Figure 1C) & 0.1 & $+30^{\circ}$ & 28 & 33.1 & 27.8 & $0 / 89$ \\
\hline 1.0 & (Top, Figure 1C) & 0.25 & $0^{\circ}$ & 26 & 61.9 & 24.0 & $8 / 100$ \\
\hline 1.0 & & 0.25 & $+30^{\circ}$ & 18 & 38.8 & 25.3 & $0 / 87$ \\
\hline 0.5 & (Top, Figure 1B) & 0.1 & $0^{\circ}$ & 18 & 79.5 & 23.1 & $0 / 100$ \\
\hline 0.5 & & 0.1 & $+30^{\circ}$ & 26 & 27.0 & 24.4 & $0 / 80$ \\
\hline 0.5 & & 0.25 & $0^{\circ}$ & 36 & 20.9 & 18.8 & $0 / 62$ \\
\hline 0.5 & & 0.25 & $+30^{\circ}$ & 28 & 24.8 & 20.1 & $0 / 80$ \\
\hline 0.25 & (Bottom, Figure 1B) & 0.1 & $0^{\circ}$ & 27 & 45.1 & 23.5 & $0 / 87$ \\
\hline 0.25 & & 0.1 & $+30^{\circ}$ & 36 & 11.8 & 11.6 & $0 / 50$ \\
\hline 0.25 & & 0.25 & $0^{\circ}$ & 26 & 12.8 & 15.8 & $0 / 75$ \\
\hline 0.25 & & 0.25 & $+30^{\circ}$ & 18 & 8.2 & 8.4 & $0 / 30$ \\
\hline \multicolumn{8}{|c|}{ "INCONSISTENT TREATMENT" PATTERN OF RESULTS } \\
\hline 1.0 & (Middle, Figure 1A) & 0.1 & $0^{\circ}$ & 36 & 17.3 & 15.3 & $0 / 75$ \\
\hline 1.0 & & 0.1 & $+30^{\circ}$ & 28 & 14.4 & 14.5 & $0 / 57$ \\
\hline 1.0 & & 0.25 & $0^{\circ}$ & 26 & 23.3 & 22.6 & $0 / 77$ \\
\hline 1.0 & & 0.25 & $+30^{\circ}$ & 18 & 15.8 & 17.5 & $0 / 67$ \\
\hline 0.5 & & 0.1 & $0^{\circ}$ & 18 & 14.4 & 20.1 & $0 / 85$ \\
\hline 0.5 & & 0.1 & $+30^{\circ}$ & 26 & 11.0 & 10.6 & $0 / 35$ \\
\hline 0.5 & & 0.25 & $0^{\circ}$ & 36 & 18.8 & 14.7 & $0 / 50$ \\
\hline 0.5 & & 0.25 & $+30^{\circ}$ & 28 & 12.6 & 10.3 & $0 / 35$ \\
\hline 0.25 & & 0.1 & $0^{\circ}$ & 28 & 14.0 & 17.2 & $0 / 72$ \\
\hline 0.25 & & 0.1 & $+30^{\circ}$ & 36 & 13.3 & 10.8 & $0 / 43$ \\
\hline 0.25 & & 0.25 & $0^{\circ}$ & 26 & 11.3 & 14.9 & $0 / 75$ \\
\hline 0.25 & & 0.25 & $+30^{\circ}$ & 17 & 7.2 & 6.0 & $0 / 16$ \\
\hline \multicolumn{8}{|c|}{ "IRREVERSIBLE EFFECT" PATTERN OF' RESULTS } \\
\hline 1.0 & (Bottom, Figure 1A) & 0.1 & $0^{\circ}$ & 36 & 23.2 & 18.4 & $0 / 57$ \\
\hline 1.0 & & 0.1 & $+30^{\circ}$ & 28 & 19.3 & 16.2 & $0 / 59$ \\
\hline 1.0 & & 0.25 & $0^{\circ}$ & 26 & 16.3 & 16.0 & $0 / 62$ \\
\hline 1.0 & & 0.25 & $+30^{\circ}$ & 17 & 17.3 & 14.0 & $0 / 50$ \\
\hline 0.5 & & 0.1 & $0^{\circ}$ & 18 & 23.5 & 21.0 & $0 / 85$ \\
\hline 0.5 & & 0.1 & $+30^{\circ}$ & 26 & 12.7 & 8.8 & $0 / 33$ \\
\hline 0.5 & & 0.25 & $0^{\circ}$ & 36 & 17.8 & 14.7 & $0 / 50$ \\
\hline 0.5 & & 0.25 & $+30^{\circ}$ & 28 & 15.8 & 12.6 & $0 / 50$ \\
\hline 0.25 & & 0.1 & $0^{\circ}$ & 28 & 18.5 & 17.7 & $0 / 83$ \\
\hline 0.25 & & 0.1 & $+30^{\circ}$ & 36 & 12.5 & 10.6 & $0 / 40$ \\
\hline 0.25 & & 0.25 & $0^{\circ}$ & 26 & 11.7 & 11.1 & $0 / 50$ \\
\hline 0.25 & & 0.25 & $+30^{\circ}$ & 18 & 9.6 & 8.3 & $0 / 25$ \\
\hline
\end{tabular}

${ }^{a} \mathrm{~N}=108$

${ }^{\text {bMaximum score }}=100$

Topography of results. Most frequently mentioned were "trends" (31) or "slopes" (9), their "relation to the specific experimental hypotheses under investigation" (6), and changes in them both within (2) and between conditions. Next indicated were "means of phases" (32) and more complicated notions of "levels," (which involved judgments about "data overlap" across conditions rather than arithmetic averages). Next in frequency were the related notions of "stability" (24) and "variability" (26), both within and across conditions. At least two judges said that variability must be expressed "relative to the variability of other subjects in the same or 
similar studies." "Replication" of effect was also an important criteria (15), with five judges stating that the "number of" replications could be basis for this decision. Four judges indicated that "replication across subjects" was important. An interesting exclusion criterion for interpreting effects was posited by two judges, namely that there be "no evidence of (unintended) sequence or cyclical effects." Three judges simply stated that effect must be "clear," "unambiguous," and "significant."

Format of presentation. Twelve judges noted that the "labeling and scaling" of both axes must bear clear relationship to the dependent and independent variables of the study, that the graph be "easily read" and "consistent with text." Thirteen felt that the "reliability of measurement," and "scope of changes therein" must be specified for accurate interpretation. Seven considered the "number of data points in each phase" as important while one each mentioned the "presence of follow-up" and the specification of the "chronology of data gathering" as desirable practices.

Intra-experimental concerns. If data on more than one subject are under investigation, variability, effects, and mean changes are judged across subjects in a relative fashion. Other reviewers stressed that their interpretations of graphs took other factors into account. "Relation to design" was mentioned by four, "nature of experimental question under investigation" by an equal number, and one each designated "conclusions of author" and "procedures" as salient factors. Two reviewers also cited as desirable a "low probability of alternative explanations or potential confounds."

Extra-experimental concerns. Some subjects took into account information that is not strictly a part of any given experiment. Paramount among these was the well-known issue of "social" (19), "clinical" (7), and/or "applied" (4) "significance" of results. Five other reviewers indicated that the question of "frequency of research previously completed" in an area was a significant concern to them, in the sense that they would be unlikely to recommend publication of an exact replication unless the control of new variables or an "enhanced" effect was evident.

\section{DISCUSSION}

The Jones et al. (1978) study compared visual with statistical inference and found that visual inference of a panel of judges was not particularly reliable. The present study replicated this finding and, in addition, attempted to assess the factors contributing to reliable or unreliable visual judgment. The median interjudge agreement correlation was a modest .61. Except for the ideal pattern of results (in the hypothesized data), a wide range of ratings of effect was obtained for almost every graph. Graphic characteristics appear to determine judgments in concert rather than singly. It seems likely that raters weigh these factors differently when processing graphic information, which is quite plausible when one considers the wide range of evaluative criteria stated.

The above variability of opinions suggests that a behavioral researcher seeking corroboration on the interpretation of results would not be likely to get the same answer twice. A statistical analysis, however, produces the same result each time which could provide a "yardstick" for comparing effects from one experiment to another (which is a judgment that many reviewers now make subjectively). Such a yardstick would also be useful in single-case studies for assessing the relative contribution of variables in which the experimental goal is to isolate causes of variability or the contribution of "indvidual differences" (Hersen and Barlow, 1976). Thus, any behavioral research effort in which the information from one graph must be related to the information in another might be conducted more efficiently with a reliable judgmental aide, statistical or visual, for operationalizing effects. 


\section{REFERENCES}

Baer, D. M. "Perhaps it would be better not to know everything." Journal of Applied Behavior Analy. sis, 1977, 10, 167-172.

Hersen, M. and Barlow, D. H. Single case experimental designs: strategies for studying behavior change. New York: Pergamon, 1976.

Jones, R. R., Weinrott, M. R., and Vaught, R. S. Effects of serial dependency on the agreement between visual and statistical inferences. Journal of Applied Behavior Analysis, 1978, 11, 277-284.

Kazdin, A. E. Statistical analysis for single case experimental designs. In $\mathbf{M}$. Hersen and D. $\mathrm{H}$. Barlow (Eds), Single case experimental designs. strategies for studying behavior change. New York: Pergamon, 1976.

Sidman, M. Tactics of scientific research. New York: Basic Books, 1960.

White, O. R. Pragmatic approaches to progress in the single case. Doctoral dissertation, University of Oregon, 1971, University Microfilms, 72-8618, Ann Arbor, Michigan.

Received 19 June 1978.

(Final Acceptance 19 July 1979.) 